\title{
Constipation: A potentially serious adverse effect of clozapine that's often overlooked
}

\section{Shahnaz Kasmani, MD}

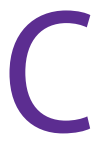

lozapine is the most effective second-generation antipsychotic for the treatment of refractory schizophrenia. It can reduce delusions and hallucinations in patients who are unresponsive to other antipsychotic medications. Further, clozapine is the only agent known to reduce suicidal urges. ${ }^{1}$

Unfortunately, clozapine is associated with numerous adverse effects, most notably agranulocytosis, a rare but potentially fatal adverse effect that occurs in approximately $1 \%$ to $2 \%$ of patients during the first year of treatment. ${ }^{2}$ Other adverse effects associated with clozapine are weight gain, sedation, orthostatic hypotension, sialorrhea, constipation, hyperglycemia, hyperlipidemia, myocarditis, and seizures. Among these adverse effects, constipation, which can progress to life-threatening gastrointestinal (GI) hypomotility and ileus, is often overlooked. Up to $60 \%$ of patients who are administered clozapine experience constipation. ${ }^{3}$ A recent review found that potentially life-threatening clozapine-induced ileus occurred in approximately 3 per 1,000 patients, and 28 deaths have been documented. ${ }^{4}$

In this case report, I describe a patient who received clozapine and experienced constipation that led to an intestinal obstruction. I discuss the importance of prompt diagnosis and treatment approaches to prevent severe constipation in patients who are prescribed clozapine.

\section{CASE REPORT}

Mr. L, age 24, has schizophrenia, depression, mild intellectual disability, and congenital

human immunodeficiency virus (HIV). He has had multiple unsuccessful antipsychotic trials but is compliant with highly active antiretroviral therapy for HIV. After experiencing worsening aggressive behavior for a third time, Mr. L was involuntarily committed to our Crises Response Center.

Mr. L was admitted to the acute inpatient psychiatry unit. He reported having auditory hallucinations, which included whispering sounds with intermittent music, mostly at night. He also reported decreased sleep, poor appetite, and low energy, but denied feelings of depression or mania.

During the mental status examination, Mr. L was calm and cooperative, but easily distracted. He said he smoked cigarettes but denied any current alcohol or illicit drug use. Mr. L's urine drug screen was negative.

External medication records showed Mr. L had been prescribed haloperidol, risperidone, chlorpromazine, olanzapine, aripiprazole, quetiapine, bupropion, sodium valproate, and topiramate, for the treatment of schizophrenia, with no significant improvement.

On hospital Day 3, Mr. L was started on clozapine, $12.5 \mathrm{mg}$ at bedtime, and titrated to $300 \mathrm{mg}$ by Day 15 . The clozapine was titrated slowly; initially the dose was doubled every 2 days up to $100 \mathrm{mg}$ every night at bedtime,

LET YOUR VOICE BE HEARD

CURRENT PSYCHIATRY invites psychiatry residents to share their views on professional or clinical topics for publication in Residents' Voices. E-mail jbauer@mdedge.com for author guidelines.

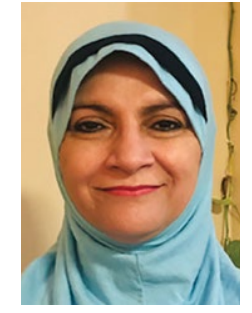

Dr. Kasmani is a PGY-3 Psychiatry Resident, Department of Psychiatry, Einstein Healthcare Network, Philadelphia, Pennsylvania. Disclosure The author reports no financial relationships with any companies whose products are mentioned in this article, or with manufacturers of competing products.

doi: $10.12788 /$ cp.0085 


\section{Clinical Point}

\section{Patients may have difficulty reporting intestinal pain due to psychotic symptoms such as paranoia or thought disorder}

Discuss this article at www.facebook.com/ MDedgePsychiatry then it was increased by $50 \mathrm{mg}$ every 2 to 3 days up to $300 \mathrm{mg}$ every night at bedtime. A baseline complete blood count with differential confirmed that his absolute neutrophil count (ANC) was $>1,500 \mu \mathrm{L}$, which is above the reference range. Mr. $L$ was closely monitored for agranulocytosis and had weekly blood work for ANC. Additionally, his information was updated regularly on the Clozapine Risk Evaluation and Mitigation Strategy website.

After Mr. $L$ began the clozapine regimen, he had reduced mood lability, paranoia, and delusions; significantly improved auditory and visual hallucinations; and reduced distress. His sleep was improved, and he appeared pleasant with clear sensorium. During this period, Mr. $L$ developed sialorrhea and was administered glycopyrrolate and prescribed diphenhydramine, as needed for sleep. Although he had been prescribed oral benztropine for extrapyramidal side effects prophylaxis, this medication was never administered to him during his stay in the hospital. He became stable on this regimen, and the treatment team started working on his discharge.

On hospital Day 20, Mr. L complained about abdominal pain. At first, the pain was localized to right upper quadrant; later, he had diffuse abdominal pain with distension. He reported that he had no bowel movement for 1 day. The treatment team instructed him to take nothing by mouth, and all antipsychotic and anticholinergic medications were held. Given Mr. L's HIV status, the treatment team ordered liver function tests (LFTs) and an abdominal $x$-ray. Mr. L's LFT results were normal and the $x$-ray findings were inconclusive. However, a CT scan of the abdomen showed an obstruction due to a $3.5-\mathrm{cm}$ stoolball in the proximal transverse colon with fecal impaction. Mr. L was started on a saline enema, which resulted in him having 2 to 3 episodes of watery diarrhea, and his abdominal pain resolved.

Although Mr. L reported feeling better and started eating again, there were concerns about his watery bowel movement, so a repeat abdominal $x$-ray was ordered. The $x$-ray confirmed that Mr. L had a persis- tent bowel obstruction. Mr. L's abdominal pain returned. At this time, the pain was diffuse and severe, and Mr. $L$ was vomiting. Mr. L was started on a bisacodyl suppository immediately, and then twice daily as needed. Subsequently, Mr. L had a solid bowel movement and relief of all Gl symptoms. Mr. L was administered docusate sodium twice daily. Repeated x-rays of the abdomen confirmed the obstructive changes of the small bowel had resolved.

\section{Why constipation may be overlooked}

Although constipation is a common adverse effect of many psychotropic medications, when it emerges during clozapine therapy, it can lead to ileus, which can be fatal. Mr. L's case highlighted that clozapine use can cause intestinal obstruction, a condition that can deteriorate within a few hours to life-threatening ileus. The extent of fecal impaction can be masked by spurious diarrhea, as illustrated in Mr. L's case. ${ }^{5}$ Clozapine has anti-serotonergic properties (5HT-2A antagonist) that may result in reduced intestinal nociception pain. This discrepancy between physical symptoms and the severity of illness may cause delays in diagnosis. ${ }^{4}$ As soon as the treatment team determined Mr. L was constipated, all medications with anticholinergic effects were held. Patients also may have difficulty reporting intestinal pain due to psychotic symptoms such as paranoia or thought disorder. ${ }^{6}$

\section{Take steps to prevent constipation}

To prevent constipation in patients receiving clozapine, minimize the use of systemic anticholinergic agents because of the adverse effects of this interaction. For example, in Mr. L's case, he received both clozapine and glycopyrrolate. In addition, all patients who are prescribed clozapine should receive docusate sodium to prevent constipation. However, because docusate 
sodium alone is usually not sufficient, consider adding another agent. Osmotic laxatives, such as polyethylene glycol 3350, are suitable additional agents. If this combination does not work, then consider senna glycoside or bisacodyl, which will increase intestinal motility and help with the flow of water into the bowel, thereby improving constipation. Bulk agents should be avoided because they can make constipation worse, especially if the patient is not drinking enough water, which is often the case with patients who have psychosis. ${ }^{7}$

\section{Ask patients about Gl symptoms}

Clinicians need to observe and monitor patients who receive clozapine for signs of constipation, including the frequency and difficulty of defecation during treatment. ${ }^{4}$ It is important to ask patients about bowel function. Before starting treatment with clozapine, discuss the risks of clozapine-induced intestinal obstruction with patients and caregivers, and encourage them to report any GI symptoms. Also, provide dietary advice and recommend the as-needed use of laxatives.

\section{References}

1. Patchan KM, Richardson C, Vyas G, et al. The risk of suicide after clozapine discontinuation: cause for concern. Ann Clinical Psychiatry. 2015;27(4):253-256.

2. Alvir JM, Lieberman JA, Safferman AZ, et al. Clozapineinduced agranulocytosis. Incidence and risk factors in the United States. N Engl J Med. 1993;329(3):162-167.

3. Hayes G, Gibler B. Clozapine-induced constipation. Am J Psychiatry. 1995;152(2):298.

4. Palmer SE, McLean RM, Ellis PM, et al. Life-threatening clozapine-induced gastrointestinal hypomotility: an analysis of 102 cases. J Clin Psychiatry. 2008;69(5):759-768.

5. Drew L, Herdson P. Clozapine and constipation: a serious issue. Aust N Z J Psychiatry. 1997; 31(1):149-150.

6. Bickerstaff LK, Harris SC, Leggett RS, et al. Pain insensitivity in schizophrenic patients: a surgical dilemma. Arch Surg. 1988;123(1):49-51.

7. Psychopharmacology Institute. How to manage adverse effects of clozapine - Part 1. Updated June 3, 2020. Accessed December 7, 2020. https://psychopharmacologyinstitute. com/publication/how-to-manage-adverse-effects-ofclozapine-part-1-2476

\section{Clinical Point}

\section{To prevent} constipation in patients receiving clozapine, minimize the use of systemic anticholinergic agents 